\title{
Correction to: Projection of Iron Ore Production
}

\author{
Steve Mohr, ${ }^{1,5}$ Damien Giurco, ${ }^{1}$ Mohan Yellishetty, ${ }^{2}$ James Ward, ${ }^{3}$ and Gavin Mudd ${ }^{4}$
}

Published online: 20 November 2019

\section{Correction to:}

Natural Resources Research (2015) 24:317-327

https://doi.org/10.1007/s11053-014-9256-6

The original version of this article unfortunately contained an error in Equation 3. This error does not affect the results of the paper; merely the description of the model was incorrect. Both equa- tions are nearly identical in terms of their functionality.

The correct equation is given below:

$L_{M}(t)=\left\{\begin{array}{lc}L_{H}+\left(L_{L}-L_{H}\right) \frac{\log _{10} M_{P}(t) / M_{H}}{\log _{10} M_{L} / M_{H}} ; & \text { if } M_{L} \neq M_{H} \\ \frac{\left(L_{L}+L_{H}\right)}{2} ; & \text { otherwise }\end{array}\right.$

${ }^{1}$ Institute for Sustainable Futures, University of Technology, Sydney, Ultimo NSW 2007, Australia.

${ }^{2}$ Division of Mining \& Resources Engineering, Department of Civil Engineering, Monash University, Wellington Road, Clayton VIC 3800, Australia.

${ }^{3}$ School of Natural \& Built Environments, University of South Australia, GPO Box 2471, Adelaide SA 5000, Australia.

${ }^{4}$ Department of Civil Engineering, Monash University, Wellington Road, Clayton VIC 3800, Australia.

${ }^{5}$ To whom correspondence should be addressed; e-mail: Steve.Mohr@uts.edu.au 